\title{
Exponential Synchronization of a Class of $N$-Coupled Complex Partial Differential Systems with Time-Varying Delay
}

\author{
Wenhua Xia, ${ }^{1}$ Yiping Luo, ${ }^{1}$ Bifeng Zhou, ${ }^{2}$ and Guanghui Liu ${ }^{1}$ \\ ${ }^{1}$ College of Science, Hunan Institute of Engineering, Hunan 411104, China \\ ${ }^{2}$ Hunan Electrical College of Technology, Hunan 411101, China \\ Correspondence should be addressed to Yiping Luo; lyp@hnie.edu.cn
}

Received 1 April 2017; Revised 28 August 2017; Accepted 7 September 2017; Published 16 October 2017

Academic Editor: Pietro De Lellis

Copyright (c) 2017 Wenhua Xia et al. This is an open access article distributed under the Creative Commons Attribution License, which permits unrestricted use, distribution, and reproduction in any medium, provided the original work is properly cited.

\begin{abstract}
This paper is concerned with the exponential synchronization for a class of $N$-coupled complex partial differential systems (PDSs) with time-varying delay. The synchronization error dynamic of the PDSs is defined in the $q$-dimensional spatial domain. To achieve synchronization, we added a linear feedback controller. A sufficient condition is derived to ensure the exponential synchronization of the proposed networks using the Lyapunov-Krasovskii stability approach and matrix inequality technology. The proposed system has broad applications. Two example applications are presented in the final section of this paper to verify the proposed theoretical result.
\end{abstract}

\section{Introduction}

Over the last few years, complex dynamical networks have been used to describe numerous large-scale systems in different fields, such as natural and human societies. A complex dynamical network is a large set of interconnected nodes; each node represents an individual in the system, whereas the edges denote the relations between them. Typical examples include physical systems, biological neural networks, the Internet, electrical power grids, and social networks. Many interesting and important studies have been previously conducted on various complex dynamical networks [1-5].

Synchronization, a common phenomenon in real systems, occurs within widespread fields such as flushing fireflies, brain web, distributed computing systems, sensor networks, and applause and ranges from natural to artificial networks. The present experiment proves the following: the flicker frequency of a firefly is affected by the flicker frequency of its surrounding luminescence; heart muscle cells can relax and contract the heart valve through synchronous oscillations. Synchronizations are vital in our daily life. Thus, we must find conditions to guarantee that the nodes in a network converge on the same desired trajectory; that is, the network achieves synchronization. Therefore, the synchronization problem of complex networks has attracted great attention in the past and is becoming an important topic. Many important results on synchronization have been obtained for various complex dynamical networks [3-6]. External force controllers usually need to be designed and applied to ensure the synchronization of networks. Several control schemes, such as adaptive, impulsive, and pinning control, have been reported [7-10]. Scholars have used various methods to study asymptotical synchronization, exponential synchronization, passivity synchronization, and $H_{\infty}$ synchronization, for different complex networks, and have achieved fruitful results [11-24].

Many phenomena exist in practice, such as the ones in chemical engineering, neurophysiology, and biodynamics, where state variables depend not only on time but also on spatial position. These phenomena are generally modeled in partial differential systems (PDSs). Therefore, increasing concerns have risen on the study of PDSs [25-43]. A significant part of research is based on reaction-diffusion neural network models, such as [25, 34-38]. Multiple intercoupled reaction-diffusion neural networks can generate complex networks, as shown in the following example: the author of [25] discusses the passivity-based synchronization of a complex delayed dynamical network consisting of $N$ linearly 
and diffusively coupled identical reaction-diffusion neural networks. In practice, the nodes of a complex network are not necessarily neurons. For example, in [26], Yang et al. address a class of complex spatiotemporal networks with space-varying coefficients, where node dynamics are described by coupled partial differential equations (PDEs); in [27], Wang et al. study a class of networked linear spatiotemporal networks consisting of $N$ identical nodes, where the spatiotemporal behavior of each node is described by parabolic PDEs. Complex network systems with a partial differential term are generally studied more.

The synchronization of complex partial differential systems has also yielded fruitful research results [25-29, 3943]. Some of the studies have been based on networks without time delay, in which the spatial variables are onedimensional, such as [26-28]. However, the spatial variable $x$ is not always one-dimensional. Thus, the authors expand spatial variables into the $q$-dimensional $(q \geq 2)$ spatial domain to discuss asymptotical [39], $H_{\infty}$ [39], and finite-time synchronizations [40] for two PDSs. No delay exists in the networks in the literature mentioned earlier.

Time delays are often encountered in practical cases, and their existence is often one of the key factors that cause system shock and instability. Hence, the problem of considering time delay in the PDSs has aroused the interest of researchers. In [29], sufficient conditions on asymptotical synchronization for a class of coupled time-delay PDSs via boundary control have been obtained; Wu et al. address the robust $H_{\infty}$ synchronization of PDSs with time delay [41, 42]; Wang et al. focus on the passivity problem of a class of delayed reaction-diffusion networks [43].

To date, some studies have been conducted on the exponential synchronization of PDSs, where spatial variables are one-dimensional and the networks have no time lag $[26,27]$. However, no literature on the exponential synchronization of time-delay PDS in $q$-dimensional space variables has been published.

Motivated by the above analysis, this paper considers the exponential synchronization for a class of time-varying delay $N$-coupled PDSs in the $q$-dimensional spatial domain. The innovation points of this paper can be summarized as follows:

(1) We propose a method that studies exponential synchronization for the time-delay partial differential complex network model with reaction-diffusion term presented in this paper. That is, the sufficient condition of delay-dependent exponential synchronization is obtained by constructing the appropriate Lyapunov-Krasovskii function and using matrix inequality analysis technology. The sufficient condition is presented in the form of a linear matrix inequality and can reflect the influence of time delay on system exponential synchronization, hence becoming a less conservative method than the existing models. To the best of our knowledge, the exponential synchronization of the time-delay partial differential complex network model with multidimensional spatial variables has not yet been studied.
(2) We reveal the influence of a diffusion coefficient on the exponential synchronization of the proposed system. The sufficient condition obtained reveals an interesting conclusion: if $D$ is sufficiently large, then condition (16) of Theorem 8 will always be satisfied. Hence, a given system will always reach exponential synchronization if the diffusion coefficient is large enough.

(3) For the proposed system, we can use the obtained sufficient condition of exponential synchronization and the LMI Control Toolbox in MATLAB to easily calculate the estimated values of the maximum delay margin $\delta$ and maximum exponential synchronization decay rate $\mu_{0}$.

The rest of this paper is organized as follows. In Section 2, a class of $N$-coupled complex PDSs with time-varying delay is presented and some preliminaries are given. In Section 3, we analyze the synchronization of the addressed network based on the Lyapunov-Krasovskii stability theorem and linear matrix inequalities (LMIs) technology. Two numerical examples are provided to verify the effectiveness of the theoretical results in Section 4. Section 5 presents the concluding remarks.

\section{Network Model and Preliminaries}

2.1. Notations. Throughout this paper, $R^{n}$ and $R^{n \times m}$ denote the $n$-dimensional Euclidean space and the set of all $n \times$ $m$ real matrices, respectively. For symmetric matrix $A$, the notation $A>0$ means that $A$ is a positive-definite matrix. $\lambda_{\text {min }}(\cdot)$ and $\lambda_{\max }(\cdot)$ represent the minimum and maximum eigenvalues, respectively, of the matrix. $C^{s}(\Omega)$ refers to the space of functions with continuous partial derivatives of an order less than or equal to $s$ in $\Omega$. The symbol $\otimes$ denotes the Kronecker product.

2.2. Model Description. Consider the following $N$-coupled complex PDSs with time-varying delay, in which each node is an $n$-dimensional dynamical subsystem:

$$
\begin{aligned}
\frac{\partial z_{i}(x, t)}{\partial t}= & D \Delta z_{i}(x, t)+A f\left(z_{i}(x, t)\right) \\
& +C_{1} \sum_{j=1}^{N} R_{i j}^{1} \Gamma_{1} z_{j}(x, t) \\
& +C_{2} \sum_{j=1}^{N} R_{i j}^{2} \Gamma_{2} z_{j}(x, t-h(t)) .
\end{aligned}
$$
follows:

The boundary conditions and initial values are given as

$$
\begin{aligned}
& z_{i}(x, t)=\Phi_{i}(x, t) \quad(x, t) \in \Omega \times\left[-h_{0}, 0\right] \\
& z_{i}(x, t)=0 \quad(x, t) \in \partial \Omega \times\left[-h_{0},+\infty\right],
\end{aligned}
$$

where $\Phi_{i}(x, t)$ is bounded and continuous on $\Omega, i=$ $1,2, \ldots, N ; N$ is the number of nodes in the network; 
$x \in\left(x_{1}, x_{2}, \ldots, x_{q}\right)^{T} \in \Omega \subset R^{q}$ is the space variable; $z_{i}(x, t)=\left(z_{i 1}(x, t), z_{i 2}(x, t), \ldots, z_{i n}(x, t)\right)^{T} \in R^{n}$ is the state vector of the $i$ th node; $t$ is the time variable; $f\left(z_{i}\right)=\left(f_{1}\left(z_{i 1} \cdot\right), f_{2}\left(z_{i 2} \cdot\right), \ldots, f_{n}\left(z_{i n} \cdot\right)\right)^{T}$ is the smooth nonlinear vector-valued function; $C_{1}$ and $C_{2}$ are the positive real numbers for the coupling strength for nondelayed and delayed configurations, respectively; $D=\operatorname{diag}\left(d_{1}, d_{2}, \ldots, d_{n}\right)$ is the positive-definite matrix; $d_{i}>0, i=1,2, \ldots, n$, is diffusion reaction coefficients; $A=\left(a_{i j}\right)_{n \times n}$ is the real matrix; $\Gamma_{1}=\operatorname{diag}\left(\gamma_{1}{ }^{1}, \gamma_{2}{ }^{1}, \ldots, \gamma_{n}{ }^{1}\right)$ and $\Gamma_{2}=\operatorname{diag}\left(\gamma_{1}{ }^{2}, \gamma_{2}{ }^{2}, \ldots, \gamma_{n}{ }^{2}\right)$ are the positive-definite matrices describing the individual coupling between two nodes for nondelayed and delayed configurations, respectively; $R^{1}=\left(R_{i j}^{1}\right)_{N \times N}$ and $R^{2}=\left(R_{i j}^{2}\right)_{N \times N}$ are the topological network structure and coupling strength between nodes for nondelayed and delayed configurations, respectively; if a connection exists between nodes $i$ and $j(j \neq$ $i)$, then $R_{i j}^{1}=R_{j i}^{1}>0$; else, $R_{i j}^{1}=R_{j i}^{1}=0$; and the elements of the coupling matrix $R^{1}=\left(R_{i j}^{1}\right)_{N \times N}$ satisfy

$$
R_{i i}^{1}=-\sum_{j=1, j \neq i}^{N} R_{i j}^{1}<0, \quad i=1,2, \ldots, N .
$$

$R^{2}=\left(R_{i j}^{2}\right)_{N \times N}$ can be defined similarly as $R^{1}=\left(R_{i j}^{1}\right)_{N \times N}$. The network in this paper is undirected and weighted.

\subsection{Preliminaries}

Lemma 1 (see [44]). Let $\Omega$ be a cube $\left|x_{k}\right|<l_{k}(k=1,2, \ldots, q)$ and let $g(x)$ be a real-valued function belonging to $C^{1}(\Omega)$ which vanishes on the boundary $\partial \Omega$ of $\Omega$ (i.e., $\left.g(x)\right|_{\partial \Omega}=0$ ). Then,

$$
\begin{aligned}
\int_{\Omega} g^{2}(x) d x \leq l_{k}^{2} \int_{\Omega}\left(\frac{\partial g(x)}{\partial x_{k}}\right)^{2} d x & \\
\text { where } x & \in\left(x_{1}, x_{2}, \ldots, x_{q}\right)^{T} .
\end{aligned}
$$

Lemma 2 (see [16]). For any vector $x, y \in R^{n}$, scalar $\mu>0$, and positive-definite matrix $Q \in R^{n \times n}$, the following inequality holds:

$$
2 x^{T} y \leq \mu x^{T} Q x+\frac{1}{\mu} y^{T} Q^{-1} y .
$$

Lemma 3 (Schur complement). Suppose $E_{1}$ and $E_{2}$ are symmetric matrices; the linear matrix inequality (LMI)

$$
\left(\begin{array}{cc}
E_{1} & E_{3} \\
E_{3}{ }^{T} & E_{2}
\end{array}\right)<0
$$

is equivalent to one of the following conditions:

(1) $E_{1}<0, E_{2}-E_{3}{ }^{T} E_{1}{ }^{-1} E_{3}<0$,

(2) $E_{2}<0, E_{1}-E_{3} E_{2}{ }^{-1} E_{3}^{T}<0$,

where $E_{3}$ is a matrix with suitable dimensions.
Assumption 4. For any vector $x, y \in R^{n}$, there exists a positive constant $\rho_{j} \in R$ such that the function $f_{j}(\bullet)$ satisfies the Lipschitz condition

$$
\left|f_{j}(x)-f_{j}(y)\right| \leq \rho_{j}|x-y| .
$$

Assumption 5. There exist real positive constants $h_{0}$ such that the time delay $h(t)$ satisfies $\dot{h}(t) \leq h_{0}<1$.

Let $s(x, t)=\left(s_{1}(x, t), s_{2}(x, t), \ldots, s_{n}(x, t)\right)^{T}$ be the function to which all $z_{i}(i=1,2, \ldots, N)$ are expected to synchronize and $s(x, t)$ satisfies the following equation:

$$
\begin{aligned}
\frac{\partial s}{\partial t} & =D \Delta s(x, t)+A f(s(x, t)), \\
s(x, t) & =0, \quad x \in \partial \Omega, \\
s(x, t) & =\Phi(x, t), \quad(x, t) \in \Omega \times\left[-h_{0}, 0\right],
\end{aligned}
$$

where $s(x, t)$ may be an equilibrium point, a periodic orbit, or a chaotic orbit in the phase space and $\Phi(x, t)$ is bounded and continuous on $\Omega$.

Definition 6. The complex network (1) with initial condition (2) is said to achieve exponential synchronization if there exist constants $\mu>0, M>0$ such that

$$
\left\|z_{i}(x, t)-s(x, t)\right\|_{2}=M \exp \{-\mu t\}
$$

$$
\forall, i=1,2, \ldots, N \text {, }
$$

where $\|\cdot\|_{2}$ stands for the Euclidean vector norm and $s(x, t)$ is synchronous evolution of network (1).

Remark 7. Notice that $\left\|g_{i}(x, t)\right\|_{2}=\left(\int_{\Omega}\left|g_{i}(x, t)\right|^{2} d x\right)^{1 / 2}$, where $g(x, t)=\left(g_{1}(x, t), g_{2}(x, t), \ldots, g_{n}(x, t)\right)^{T}$; then,

$$
\begin{aligned}
\|g(x, t)\|_{2}^{2} & =\sum_{i=1}^{n}\left\|g_{i}(x, t)\right\|_{2}^{2}=\sum_{i=1}^{n}\left(\int_{\Omega}\left|g_{i}(x, t)\right|^{2} d x\right) \\
& =\int_{\Omega} g^{T} g d x .
\end{aligned}
$$

We add linear state feedback controllers to nodes in network (1); the controlled network is then described by

$$
\begin{aligned}
\frac{\partial z_{i}(x, t)}{\partial t}= & D \Delta z_{i}(x, t)+A f\left(z_{i}(x, t)\right) \\
& +C_{1} \sum_{j=1}^{N} R_{i j}^{1} \Gamma_{1} z_{j}(x, t) \\
& +C_{2} \sum_{j=1}^{N} R_{i j}^{2} \Gamma_{2} z_{j}(x, t-h(t))+u_{i}(x, t), \\
& i=1,2, \ldots, N,
\end{aligned}
$$

where $u_{i}(x, t), i=1,2, \ldots, N$, are the linear state feedback controllers. And $u(x, t)=\left(u_{1}{ }^{T}(x, t), u_{2}{ }^{T}(x, t), \ldots, u_{n}{ }^{T}(x, t)\right)^{T}$ is defined by

$$
u(x, t)=-C_{1} K\left(z(x, t)-s^{*}(x, t)\right),
$$


where $s^{*}(x, t)=\left(s^{T}(x, t), s^{T}(x, t), \ldots, s^{T}(x, t)\right)^{T}, K \quad \in$ $R^{n N \times n N}$.

Let $e_{i}(x, t)=z_{i}(x, t)-s(x, t)$, and error dynamics can be described as follows:

$$
\begin{aligned}
\frac{\partial e_{i}(x, t)}{\partial t}= & D \Delta e_{i}(x, t)+A f\left(z_{i}(x, t)\right)-A f(s(x, t)) \\
& +C_{1} \sum_{j=1}^{N} R_{i j}^{1} \Gamma_{1} e_{j}(x, t) \\
& +C_{2} \sum_{j=1}^{N} R_{i j}^{2} \Gamma_{2} e_{j}(x, t-h(t))+u_{i}(x, t) .
\end{aligned}
$$

The synchronization problem of PDSs in (11) becomes the stabilization problem in (13) of the corresponding synchronization.

We can rewrite system (13) in a compact form by

$$
\begin{aligned}
\frac{\partial e(x, t)}{\partial t}= & \bar{D} \Delta e(x, t)+\bar{A} \hat{f}(z(x, t))-\bar{A} \hat{f}(s(x)) \\
& +C_{1}\left(R^{1} \otimes \Gamma_{1}\right) e(x, t) \\
& +C_{2}\left(R^{2} \otimes \Gamma_{2}\right) e(x, t-h(t)) \\
& -C_{1} \operatorname{Ke}(x, t),
\end{aligned}
$$

where $\widehat{f}(z(x, t))=\left(f^{T}\left(z_{1}(x, t)\right), f^{T}\left(z_{2}(x, t)\right), \ldots, f^{T}\left(z_{N}(x\right.\right.$, $t)))^{T}, e(x, t)=\left(e_{1}^{T}(x, t), e_{2}^{T}(x, t), \ldots, e_{N}^{T}(x, t)\right)^{T}$.

Denote

$$
\begin{aligned}
& \Theta=\operatorname{diag}\left(\rho_{1}^{2}, \rho_{2}^{2}, \ldots, \rho_{n}^{2}\right), \\
& \bar{\Theta}=I_{N} \otimes \Theta, \\
& \bar{D}=\operatorname{diag}(D, D, \ldots, D)=I_{N} \otimes D, \\
& \bar{A}=\operatorname{diag}(A, A, \ldots, A)=I_{N} \otimes A .
\end{aligned}
$$

\section{Main Results}

Theorem 8. Suppose Assumptions 4 and 5 hold for the complex dynamical network (1) with initial condition (2), $0<$ $h(t) \leq \delta$, if the following matrices exist: $K \in R^{n N \times n N}, P=$ $\operatorname{diag}\left\{P^{1}, P^{2}, \ldots, P^{N}\right\}\left(\in R^{n N \times n N}\right)>0, P^{i}=\left(p_{j l}^{i}\right)_{n \times n}, Q(\epsilon$ $\left.R^{n N \times n N}\right)>0$, and $i=1,2, \ldots, N$,

$$
\left(\begin{array}{cc}
E_{1} & E_{3} \\
E_{3}{ }^{T} & -Q
\end{array}\right)<0,
$$

where

$$
\begin{aligned}
& E_{3}{ }^{T}=\exp \{-\mu \delta\} C_{2} P\left(R^{2} \otimes \Gamma_{2}\right) \\
& E_{1}=\exp \{-2 \mu \delta\}\left[-\sum_{k=1}^{q} \frac{1}{l_{k}^{2}}(P \bar{D}+\bar{D} P)+\bar{\Theta}\right.
\end{aligned}
$$

$$
\begin{aligned}
& +P \bar{A} \bar{A}^{T} P+C_{1} P\left(R^{1} \otimes \Gamma_{1}\right)+C_{1}\left(R^{1} \otimes \Gamma_{1}\right) P \\
& \left.+\frac{Q \exp \{2 \mu \delta\}}{1-h_{0}}-\left(K^{T} P+P K\right)+2 \mu P\right]
\end{aligned}
$$

and network (1) is then exponentially synchronized under controllers (12) in Definition 6.

Proof. Construct the following Lyapunov functional:

$$
\begin{aligned}
& V(t)=V_{1}+V_{2}, \\
& V_{1}=\exp \{-2 \mu \delta\} \int_{\Omega} e^{T}(x, t) \operatorname{Pe}(x, t) d x, \\
& V_{2}=\frac{1}{1-h_{0}} \int_{t-h(t)}^{t} \int_{\Omega} e^{T}(x, s) Q e(x, s) \\
& \cdot \exp \{-2 \mu(t-s)\} d x d s .
\end{aligned}
$$

From Assumption 5, the time derivative of $V(t)$ along the trajectory of system (13) is as follows:

$$
\begin{aligned}
& \dot{V}(t)=\exp \{-2 \mu \delta\} \int_{\Omega}\left(\frac{\partial e^{T}(x, t)}{\partial t} P e(x, t)+e^{T}(x, t)\right. \\
& \left.P \frac{\partial e(x, t)}{\partial t}\right) d x+\frac{1}{1-h_{0}} \int_{\Omega} e^{T}(x, t) Q e(x, \\
& t) d x-\frac{1-\dot{h}(t)}{1-h_{0}} \exp \{-2 \mu h(t)\} \int_{\Omega} e^{T}(x, t \\
& -h(t)) Q e(x, t-h(t)) d x-2 \mu V_{2} \leq \exp \{-2 \mu \delta\} \\
& \cdot \int_{\Omega}\left[e^{T}(x, t) P \bar{D} \Delta e(x, t)+(\Delta e(x, t))^{T} \bar{D} P e(x, t)\right. \\
& +e^{T}(x, t)\left(C_{1} P\left(R^{1} \otimes \Gamma_{1}\right)+\frac{Q \exp \{2 \mu \delta\}}{1-h_{0}}\right. \\
& \left.+C_{1}\left(R^{1} \otimes \Gamma_{1}\right) P\right) e(x, t)+2 e^{T}(x, t) \\
& \text { - } P \bar{A}(\widehat{f}(z(x, t))-\widehat{f}(s(x)))-e^{T}(x, t)\left(K^{T} P\right. \\
& +P K) e(x, t)+2 C_{2} e^{T}(x, t) P\left(R^{2} \otimes \Gamma_{2}\right) e(x, t \\
& -h(t))] d x-\exp \{-2 \mu \delta\} \int_{\Omega} e^{T}(x, t-h(t)) \\
& \text { - } Q e(x, t-h(t)) d x-2 \mu V_{2} \text {. }
\end{aligned}
$$

By using Green's formula and the boundary condition (2), from Lemma 1, we can obtain the following:

$$
\begin{aligned}
\int_{\Omega} e^{T}(x, t) P \bar{D} \Delta e(x, t) d x \\
\quad=\sum_{i=1}^{N} \int_{\Omega} e_{i}^{T}(x, t) P^{i} D \Delta e_{i}(x, t) d x
\end{aligned}
$$




$$
\begin{aligned}
& =\sum_{i=1}^{N} \sum_{j=1}^{n} \sum_{l=1}^{n} p_{j l}^{i} d_{l} \int_{\Omega} e_{i j}{ }^{T}(x, t) \Delta e_{i l}(x, t) d x \\
& =-\sum_{k=1}^{q} \sum_{i=1}^{N} \sum_{j=1}^{n} \sum_{l=1}^{n} p_{j l}^{i} d_{l} \int_{\Omega} \frac{\partial e_{i j}(x, t)}{\partial x_{k}} \frac{\partial e_{i l}(x, t)}{\partial x_{k}} d x \\
& =-\sum_{k=1}^{q} \int_{\Omega}\left(\frac{\partial e(x, t)}{\partial x_{k}}\right)^{T} P \bar{D}\left(\frac{\partial e(x, t)}{\partial x_{k}}\right) d x .
\end{aligned}
$$

Similarly,

$$
\begin{aligned}
\int_{\Omega} & (\Delta e(x, t))^{T} \bar{D} P e(x, t) d x \\
= & -\sum_{k=1}^{q} \int_{\Omega}\left(\frac{\partial e(x, t)}{\partial x_{k}}\right)^{T} \bar{D} P\left(\frac{\partial e(x, t)}{\partial x_{k}}\right) d x .
\end{aligned}
$$

We can then obtain

$$
\begin{gathered}
\int_{\Omega}\left[e^{T}(x, t) P \bar{D} \Delta e(x, t)+(\Delta e(x, t))^{T} \bar{D} P e(x, t)\right] d x \\
=-\sum_{k=1}^{q} \int_{\Omega}\left(\frac{\partial e(x, t)}{\partial x_{k}}\right)^{T}(P \bar{D}+\bar{D} P)\left(\frac{\partial e(x, t)}{\partial x_{k}}\right) d x \\
\leq \int_{\Omega} e^{T}(x, t)\left[-\sum_{k=1}^{q} \frac{1}{l_{k}^{2}}(P \bar{D}+\bar{D} P)\right] e(x, t) d x,
\end{gathered}
$$

where a real matrix $H$ exists, such that $P \bar{D}+\bar{D} P=H^{T} H$.

By using Assumption 4 and Lemma 2, we can derive

$$
\begin{aligned}
& 2 e^{T}(x, t) P \bar{A}(\hat{f}(z(x, t))-\widehat{f}(s(x))) \\
&= 2 \sum_{i=1}^{N} e_{i}^{T}(x, t) P^{i} A\left(f\left(z_{i}(x, t)\right)-f(s(x))\right) \\
& \leq \sum_{i=1}^{N} e_{i}^{T}(x, t) P^{i} A A^{T} P^{i} e_{i}(x, t) \\
&+\sum_{i=1}^{N} e_{i}^{T}(x, t) \Theta e_{i}(x, t) \\
&= e^{T}(x, t)\left(P \bar{A} \bar{A}^{T} P+\bar{\Theta}\right) e(x, t), \\
& 2 e^{T}(x, t) P\left(R^{2} \otimes \Gamma_{2}\right) e(x, t-h(t)) \\
& \leq C_{2} e^{T}(x, t) P\left(R^{2} \otimes \Gamma_{2}\right) Q^{-1}\left(R^{2} \otimes \Gamma_{2}\right) P e(x, t) \\
& \quad+\frac{1}{C_{2}} e^{T}(x, t-h(t)) Q e(x, t-h(t)) .
\end{aligned}
$$

Thus, substituting inequalities (22)-(26) in (21), we can obtain

$$
\begin{array}{r}
\dot{V}(t) \leq \exp \{-2 \mu \delta\} \int_{\Omega} e^{T}(x, t)\left[-\sum_{k=1}^{q} \frac{1}{l_{k}^{2}}(P \bar{D}+\bar{D} P)\right. \\
+\Theta+P \bar{A} \bar{A}^{T} P+C_{1} P\left(R^{1} \otimes \Gamma_{1}\right)+C_{1}\left(R^{1} \otimes \Gamma_{1}\right) P
\end{array}
$$

$$
\begin{aligned}
& +\frac{Q \exp \{2 \mu \delta\}}{1-h_{0}}+C_{2}{ }^{2} P\left(R^{2} \otimes \Gamma_{2}\right) Q^{-1}\left(R^{2} \otimes \Gamma_{2}\right) P \\
& \left.-\left(K^{T} P+P K\right)\right] e(x, t) d x-2 \mu V_{2} .
\end{aligned}
$$

Add $2 \mu V(t)$ to both sides of (27); then,

$$
\begin{aligned}
& \dot{V}(t)+2 \mu V(t) \leq \exp \{-2 \mu \delta\} \int_{\Omega} e^{T}(x, t) \\
& \quad\left[-\sum_{k=1}^{q} \frac{1}{l_{k}{ }^{2}}(P \bar{D}+\bar{D} P)+\Theta+P \bar{A} \bar{A}^{T} P\right. \\
& \quad+C_{1} P\left(R^{1} \otimes \Gamma_{1}\right)+C_{1}\left(R^{1} \otimes \Gamma_{1}\right) P+\frac{Q \exp \{2 \mu \delta\}}{1-h_{0}} \\
& \quad+C_{2}{ }^{2} P\left(R^{2} \otimes \Gamma_{2}\right) Q^{-1}\left(R^{2} \otimes \Gamma_{2}\right) P-\left(K^{T} P+P K\right) \\
& \quad+2 \mu P] e(x, t) d x .
\end{aligned}
$$

By using Lemma 3, from (16), we derive

$$
\dot{V}(t) \leq-2 \mu V(t) .
$$

To integrate both sides of inequality (29), we can obtain

$$
V(t) \leq \exp \{-2 \mu t\} V(0) \text {. }
$$

Given that $P>0, V(t)$, as given by (18), satisfies the following inequality:

$$
\begin{aligned}
& \exp \{-2 \mu \delta\} \lambda_{\min }(P)\|e(x, t)\|^{2} \leq V(t) \leq \exp \{-2 \mu t\} \\
& \cdot V(0) \leq \exp \{-2 \mu t\}\left[\exp \{-2 \mu \delta\} \lambda_{\max }(P)\right. \\
& \left.+\frac{1-\exp \{-2 \mu \delta\}}{2 \mu\left(1-h_{0}\right)} \lambda_{\max }(Q)\right]\|\Phi\|^{2} .
\end{aligned}
$$

Finally, we obtain

$$
\begin{aligned}
& \|e(x, t)\| \leq \exp \{-\mu t\} \\
& \cdot \sqrt{\frac{\lambda_{\max }(P)+\left((\exp \{2 \mu \delta\}-1) / 2 \mu\left(1-h_{0}\right)\right) \lambda_{\max }(Q)}{\lambda_{\min }(P)}}\|\Phi\|,
\end{aligned}
$$

where $\|\Phi\|=\sup _{-\delta \leq t \leq 0}\left[\int_{\Omega}|e(x, t)|^{2} d x\right]^{1 / 2}$.

Therefore, by using Definition 6, the error dynamical system (14) is globally exponentially stable at the equilibrium set with the exponential rate $\mu$. Consequently, network (1) is globally exponentially synchronized under controllers (12). Thus, the proof is completed.

Remark 9. To date, some studies have been conducted on the exponential synchronization of PDSs, where spatial variables are one-dimensional and the networks have no time lag [26, 27]. However, no literature on the exponential synchronization of time-delay PDS in $q$-dimensional space variables has been published. 
Remark 10. We reveal the influence of a diffusion coefficient on the exponential synchronization of the proposed system in Theorem 8 . The sufficient condition obtained reveals an interesting conclusion: if $D$ is sufficiently large, then condition (16) of Theorem 8 will always be satisfied. Hence, a given system will always reach exponential synchronization if the diffusion coefficient is large enough.

Remark 11. The criterion given in Theorem 8 is dependent on the time delay. It is well known that the delay-dependent criteria are less conservative than delay-independent criteria when the delay is small.

Remark 12. From Theorem 8, one can determine an upper bound of $\mu$ such that system (1) is exponentially synchronized. This requires solving the following optimization problem: maximize $\mu$ subject to LMI (16).

This means that system (1) under controllers (12) will be exponentially synchronized if $\mu \leq \mu_{0}$, where $\mu_{0}$ is the maximized value of $\mu$ of the optimization problem.

Remark 13. By iteratively solving the LMIs given in Theorem 8 with respect to $\delta$ and $h_{0}$, one can find the maximum allowable upper bound $\delta, h_{0}$ of $h(t)$ and $\dot{h}(t)$, respectively, for guaranteeing the exponential synchronization of system (1).

\section{Illustrative Example}

In this section, two numerical examples are provided to illustrate the effectiveness of the proposed method.

Example 1. Consider the controlled delayed PDSs consisting of four identical coupling nodes, wherein each node is a onedimensional value described by

$$
\begin{aligned}
\frac{\partial z_{i}(x, t)}{\partial t}= & D \Delta z_{i}(x, t)+A f\left(z_{i}(x, t)\right) \\
& +C_{1} \sum_{j=1}^{4} R_{i j}^{1} \Gamma_{1} z_{j}(x, t) \\
& +C_{2} \sum_{j=1}^{4} R_{i j}^{2} \Gamma_{2} z_{j}(x, t-h(t))+u_{i}(x, t) \\
& (j=1,2,3,4)
\end{aligned}
$$

with the initial values

$$
\begin{aligned}
\phi_{1}(x, 0) & =-\exp \{2(-x+1)\}, \\
\phi_{2}(x, 0) & =0.5 \sin 0.5 x, \\
\phi_{3}(x, 0) & =\exp \{1.1 *(-x+5)\}, \\
\phi_{3}(x, 0) & =5 \sin 2 x, \\
\Omega & =\{x|| x \mid \leq 0.5\},
\end{aligned}
$$

where $f(\xi)=(|\xi+1|-|\xi-1|) / 4$ satisfies the Lipschitz condition with $\rho=1 / 2$.
Take

$$
\begin{aligned}
\Theta & =0.25, \\
D & =0.3, \\
A & =0.2, \\
\Gamma_{1} & =0.4 ; \\
\Gamma_{2} & =0.1, \\
h(t) & =0.1-0.1 e^{-t}, \\
C_{1} & =0.2, \\
C_{2} & =0.1, \\
R^{1} & =\left(\begin{array}{cccc}
-0.5 & 0.2 & 0 & 0.3 \\
0.2 & -0.6 & 0.2 & 0.2 \\
0 & 0.2 & -0.2 & 0 \\
0.3 & 0.2 & 0 & -0.5
\end{array}\right), \\
R^{2} & =\left(\begin{array}{cccc}
-0.3 & 0.1 & 0 & 0.2 \\
0.1 & -0.4 & 0.2 & 0.1 \\
0 & 0.2 & -0.2 & 0 \\
0.2 & 0.1 & 0 & -0.3
\end{array}\right) .
\end{aligned}
$$

Choosing the Lyapunov positive-definite matrices $P=$ $1.7601 I_{4}$ and $Q=1.901 I_{4}$, by iteratively solving the LMIs given in Theorem 8 with respect to $K$, we can find controller matrix $K=4.1156 I_{4}$.

Moreover, we can get the maximum allowable upper bound $h_{0}=0.9496, \delta=16.18$, and $\mu_{0}=0.85$ of time delay $\dot{h}(t), h(t)$ and exponential rate $\mu$ for guaranteeing the exponential synchronization of system (33).

Example 2. If we take $h_{0}=\delta=0.1, \mu=0.1$, with the parameters given in Example 1 and by using the LMI Toolbox in Matlab, we can find the controller matrix $K$ and the Lyapunov positive-definite matrices $P$ and $Q$ as follows:

$$
K=\left(\begin{array}{cccc}
68.3649 & 0.1993 & 0.0003 & 0.2985 \\
0.1993 & 68.2660 & 0.1983 & 0.1993 \\
0.0003 & 0.1983 & 68.6641 & 0.0003 \\
0.2985 & 0.1993 & 0.0003 & 68.3649
\end{array}\right) \text {, }
$$

$$
\begin{aligned}
& P=\left(\begin{array}{llll}
1.7601 & 0.0000 & 0.0000 & 0.0000 \\
0.0000 & 1.7601 & 0.0000 & 0.0000 \\
0.0000 & 0.0000 & 1.7601 & 0.0000 \\
0.0000 & 0.0000 & 0.0000 & 1.7601
\end{array}\right), \\
& Q=\left(\begin{array}{llll}
1.9010 & 0.0000 & 0.0000 & 0.0000 \\
0.0000 & 1.9010 & 0.0000 & 0.0000 \\
0.0000 & 0.0000 & 1.9010 & 0.0000 \\
0.0000 & 0.0000 & 0.0000 & 1.9010
\end{array}\right)
\end{aligned}
$$




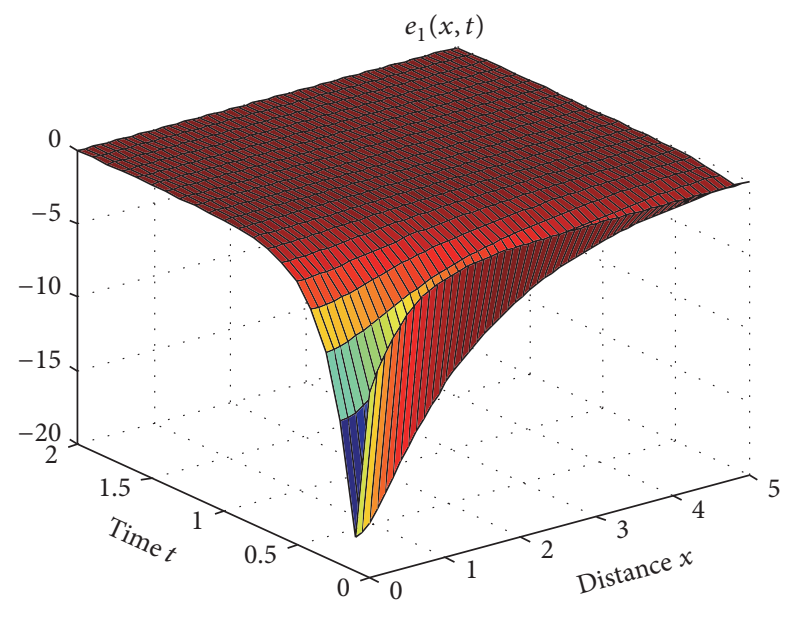

Figure 1

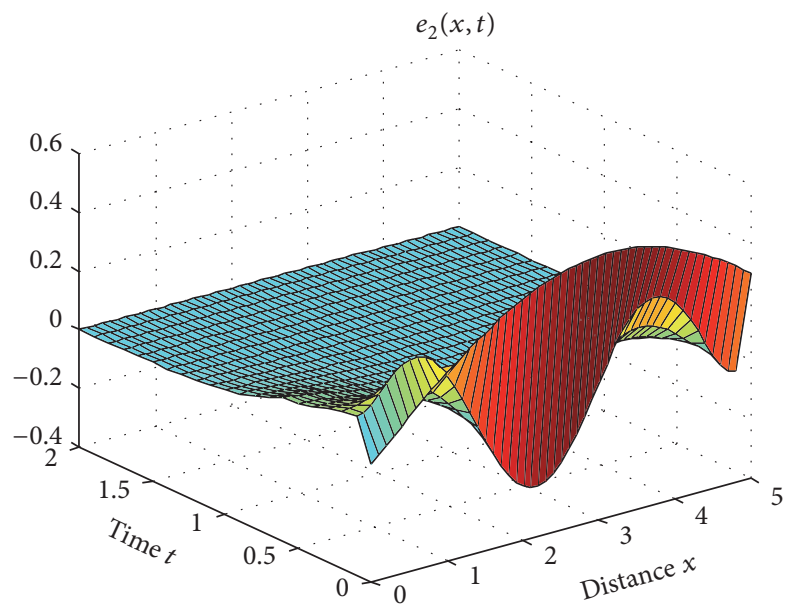

FIGURE 2

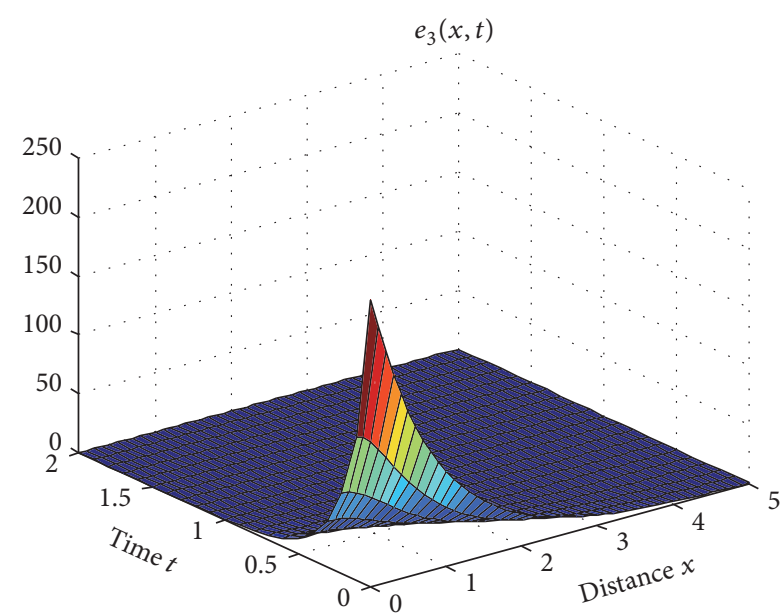

FIGURE 3

Finally, Figures 1-4 show the error dynamical system of the controlled delayed PDSs given by (33), which is globally exponentially stabilized. Network (33) is globally exponentially synchronized, which supports the proposed methods.

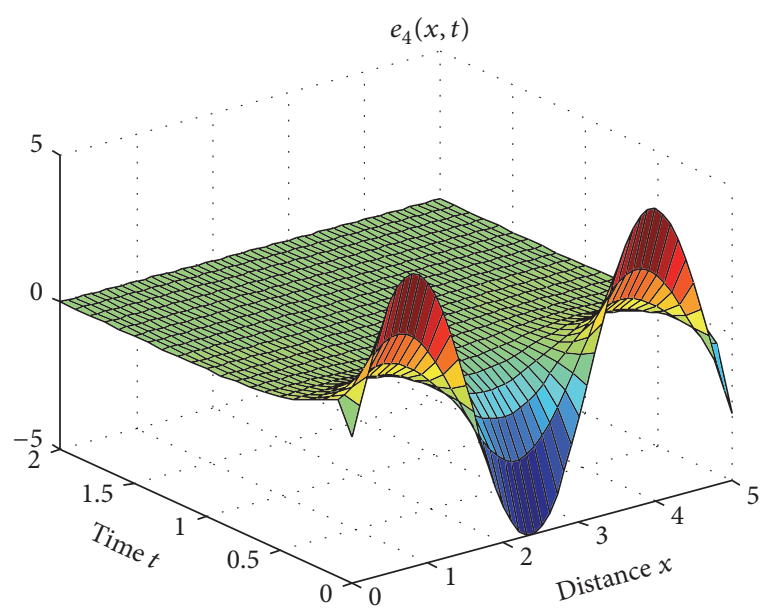

Figure 4

\section{Conclusion}

In this paper, we discuss the exponential synchronization for a class of $\mathrm{N}$-coupled complex PDSs with time-varying delay. By using Lyapunov-Krasovskii stability approach and matrix inequality technology, sufficient conditions are derived to ensure the exponential synchronization of the proposed networks. Simulations also verify our theoretical results.

\section{Conflicts of Interest}

The authors declare that they have no conflicts of interest.

\section{Acknowledgments}

This work was supported by the National Natural Science Foundation of China (11372107) and the Natural Science Foundation of Hunan Province (2017JJ4004, 2017JJ5011).

\section{References}

[1] S. H. Strogatz, "Exploring complex networks," Nature, vol. 410, no. 6825 , pp. $268-276,2001$.

[2] M. E. Newman, "The structure and function of complex networks," SIAM Review, vol. 45, no. 2, pp. 167-256, 2003.

[3] X. Wang, K. She, S. Zhong, and H. Yang, "New result on synchronization of complex dynamical networks with timevarying coupling delay and sampled-data control," Neurocomputing, vol. 214, pp. 508-515, 2016.

[4] L. Shi, H. Zhu, S. Zhong, Y. Zeng, and J. Cheng, "Synchronization for time-varying complex networks based on control," Journal of Computational and Applied Mathematics, vol. 301, pp. 178-187, 2016.

[5] R. K. Kingaid, N. Alexandrov, and M. J. Holroyd, "An Investigation of Synchrony in Transport Networks," Complexity, vol. 14, no. 4, pp. 34-43, 2009.

[6] T. Liu, G. M. Dimirovski, and J. Zhao, "Exponential synchronization of complex delayed dynamical networks with general topology," Physica A: Statistical Mechanics and its Applications, vol. 387, no. 2-3, pp. 643-652, 2008. 
[7] G. Zhang, Z. Liu, and Z. Ma, "Synchronization of complex dynamical networks via impulsive control," Chaos. An Interdisciplinary Journal of Nonlinear Science, vol. 17, no. 4, Article ID 043126, 043126, 9 pages, 2007.

[8] J. Lu, X. Wu, X. Han, and J. Lü, "Adaptive feedback synchronization of a unified chaotic system," Physics Letters, Section A: General, Atomic and Solid State Physics, vol. 329, no. 4-5, pp. 327-333, 2004.

[9] T. Chen, X. Liu, and W. Lu, "Pinning complex networks by a single controller," IEEE Transactions on Circuits and Systems. I. Regular Papers, vol. 54, no. 6, pp. 1317-1326, 2007.

[10] W. Yu, G. Chen, and J. Lü, "On pinning synchronization of complex dynamical networks," Automatica. A Journal of IFAC, the International Federation of Automatic Control, vol. 45, no. 2, pp. 429-435, 2009.

[11] Y.-P. Zhao, P. He, H. Saberi Nik, and J. Ren, "Robust adaptive synchronization of uncertain complex networks with multiple time-varying coupled delays," Complexity, vol. 20, no. 6, pp. 4960, 2015.

[12] M. A. A. Ahmed, Y. Liu, W. Zhang, and F. E. Alsaadi, "Exponential synchronization via pinning adaptive control for complex networks of networks with time delays," Neurocomputing, vol. 225, pp. 198-204, 2017.

[13] J. Wang, J. Feng, C. Xu, Y. Zhao, and J. Feng, "Pinning synchronization of nonlinearly coupled complex networks with timevarying delays using M-matrix strategies," Neurocomputing, vol. 177, pp. 89-97, 2016.

[14] J.-a. Wang, "Synchronization of delayed complex dynamical network with hybrid-coupling via aperiodically intermittent pinning control," Journal of the Franklin Institute. Engineering and Applied Mathematics, vol. 354, no. 4, pp. 1833-1855, 2017.

[15] T. Yu, D. Cao, Y. Yang, S. Liu, and W. Huang, "Robust synchronization of impulsively coupled complex dynamical network with delayed nonidentical nodes," Chaos, Solitons \& Fractals, vol. 87, pp. 92-101, 2016.

[16] Z. Tang, J. Feng, and Y. Zhao, "Global synchronization of nonlinear coupled complex dynamical networks with information exchanges at discrete-time," Neurocomputing, vol. 151, no. 3, pp. 1486-1494, 2015.

[17] M. Arcak, "Certifying spatially uniform behavior in reactiondiffusion PDE and compartmental ODE systems," Automatica. A Journal of IFAC, the International Federation of Automatic Control, vol. 47, no. 6, pp. 1219-1229, 2011.

[18] L. Scardovi, M. Arcak, and E. D. Sontag, "Synchronization of interconnected systems with applications to biochemical networks: an input-output approach," Institute of Electrical and Electronics Engineers. Transactions on Automatic Control, vol. 55, no. 6, pp. 1367-1379, 2010.

[19] J. E. Slotine and W. Wang, "A study of synchronization and group cooperation using partial contraction theory," in Cooperative control, vol. 309 of Lect. Notes Control Inf. Sci., pp. 207-228, Springer, Berlin, 2005.

[20] J. Yao, Z.-H. Guan, and D. J. Hill, "Passivity-based control and synchronization of general complex dynamical networks," Automatica. A Journal of IFAC, the International Federation of Automatic Control, vol. 45, no. 9, pp. 2107-2113, 2009.

[21] H. Zhang, M. Zhao, Z. Wang, and Z. Wu, "Adaptive synchronization of an uncertain coupling complex network with time-delay," Nonlinear Dynamics. An International Journal of Nonlinear Dynamics and Chaos in Engineering Systems, vol. 77, no. 3, pp. 643-653, 2014.
[22] J. Wang, J. Feng, C. Xu, and Y. Zhao, "Exponential synchronization of stochastic perturbed complex networks with timevarying delays via periodically intermittent pinning," Communications in Nonlinear Science and Numerical Simulation, vol. 18, no. 11, pp. 3146-3157, 2013.

[23] J. Zhao, J. Lu, and X. Wu, "Pinning control of general complex dynamical networks with optimization," Science China. Information Sciences, vol. 53, no. 4, pp. 813-822, 2010.

[24] R. Cheng, M. Peng, and W. Yu, "Pinning synchronization of delayed complex dynamical networks with nonlinear coupling," Physica A. Statistical Mechanics and its Applications, vol. 413, pp. 426-431, 2014.

[25] J.-L. Wang, H.-N. Wu, and T. Huang, "Passivity-based synchronization of a class of complex dynamical networks with timevarying delay," Automatica. A Journal of IFAC, the International Federation of Automatic Control, vol. 56, pp. 105-112, 2015.

[26] C.-D. Yang, J.-L. Qiu, and H.-B. He, "Exponential synchronization for a class of complex spatio-temporal networks with space-varying coefficients," Neurocomputing, vol. 151, pp. 401407, 2015.

[27] J.-W. Wang, C.-D. Yang, and C.-Y. Sun, "Exponential synchronization for a class of networked linear parabolic PDE systems via boundary control," in Proceedings of the 2014 International Joint Conference on Neural Networks (IJCNN '14), pp. 3981-3986, July 2014.

[28] K.-N. Wu, T. Tian, and L. Wang, "Synchronization for a class of coupled linear partial differential systems via boundary control," Journal of the Franklin Institute. Engineering and Applied Mathematics, vol. 353, no. 16, pp. 4062-4073, 2016.

[29] K.-N. Wu, T. Tian, L. Wang, and W.-W. Wang, "Asymptotical synchronization for a class of coupled time-delay partial differential systems via boundary control," Neurocomputing, 2015.

[30] E. Fridman and Y. Orlov, "An LMI approach to H1 boundary control of semi linear parabolic and hyperbolic systems," Automatica, vol. 45, no. 9, pp. 2060-2066, 2009.

[31] W. He, S. Zhang, and S. S. Ge, "Adaptive boundary control of a nonlinear flexible string system," IEEE Transactions on Control Systems Technology, vol. 22, no. 3, pp. 1088-1093, 2014.

[32] F. Di Meglio, R. Vazquez, and M. Krstic, "Stabilization of a system of coupled first-order hyperbolic linear PDEs with a single boundary input," Institute of Electrical and Electronics Engineers. Transactions on Automatic Control, vol. 58, no. 12, pp. 3097-3111, 2013.

[33] H.-N. Wu, J.-W. Wang, and H.-X. Li, "Fuzzy boundary control design for a class of nonlinear parabolic distributed parameter systems," IEEE Transactions on Fuzzy Systems, vol. 22, no. 3, pp. 642-652, 2014.

[34] C. Hu, H. Jiang, and Z. Teng, "Impulsive control and synchronization for delayed neural networks with reaction-diffusion terms," IEEE Transactions on Neural Networks, vol. 21, no. 1, pp. 67-81, 2010.

[35] Q. Gan, "Exponential synchronization of stochastic neural networks with leakage delay and reaction-diffusion terms via periodically intermittent control," Chaos. An Interdisciplinary Journal of Nonlinear Science, vol. 22, no. 1, 013124, 10 pages, 2012.

[36] K. Wang, Z. Teng, and H. Jiang, "Adaptive synchronization in an array of linearly coupled neural networks with reactiondiffusion terms and time delays," Communications in Nonlinear Science and Numerical Simulation, vol. 17, no. 10, pp. 3866-3875, 2012.

[37] X. Yang, J. Cao, and Z. Yang, "Synchronization of coupled reaction-diffusion neural networks with time-varying delays 
via pinning-impulsive controller," SIAM Journal on Control and Optimization, vol. 51, no. 5, pp. 3486-3510, 2013.

[38] S.-X. Wang, Y.-L. Huang, and B.-B. Xu, "Pinning synchronization of spatial diffusion coupled reaction-diffusion neural networks with and without multiple time-varying delays," Neurocomputing, vol. 227, pp. 92-100, 2017.

[39] K. Wu and B.-S. Chen, "Synchronization of partial differential systems via diffusion coupling," IEEE Transactions on Circuits and Systems. I. Regular Papers, vol. 59, no. 11, pp. 2655-2668, 2012.

[40] J. Wang and K.-N. Wu, "Finite-time synchronization control of coupled partial differential systems," in Proceedings of the 27th Chinese Control and Decision Conference, CCDC 2015, pp. 672676, chn, May 2015.

[41] K.-N. Wu, C.-X. Li, B.-S. Chen, and Y. Yao, "Robust Ho synchronization of coupled partial differential systems with spatial coupling Delay," IEEE Transactions on Circuits and Systems II: Express Briefs, vol. 60, no. 7, pp. 451-455, 2013.

[42] K.-N. Wu, C.-X. Li, X. Wang, and B.-S. Chen, "synchronization of coupled delay partial differential systems via nonsingular transformation method," Applied Mathematical Modelling, vol. 39, no. 16, pp. 4646-4654, 2015.

[43] J.-L. Wang and H.-N. Wu, "Passivity of delayed reactiondiffusion networks with application to a food web model," Applied Mathematics and Computation, vol. 219, no. 24, pp. 11311-11326, 2013.

[44] J. G. Lu, "Global exponential stability and periodicity of reaction-diffusion delayed recurrent neural networks with Dirichlet boundary conditions," Chaos, Solitons \& Fractals, vol. 35, no. 1, pp. 116-125, 2008. 


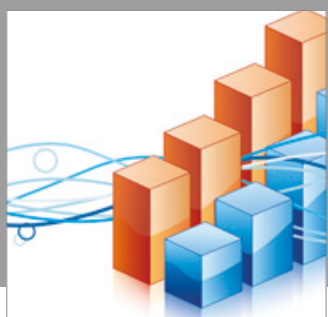

Advances in

Operations Research

vatersals

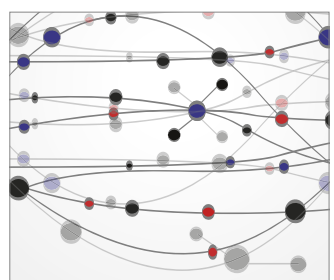

\section{The Scientific} World Journal
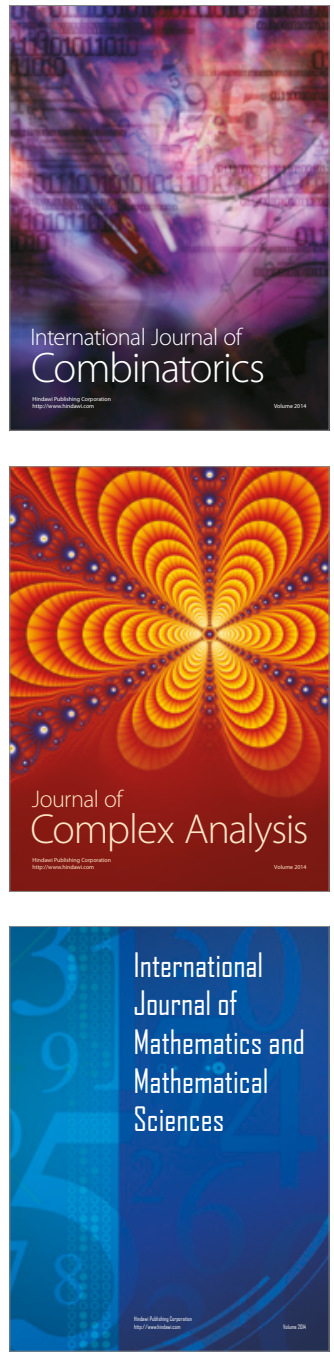
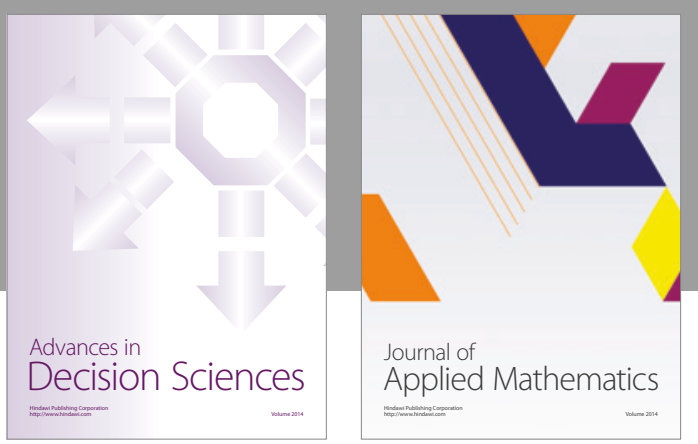

Algebra

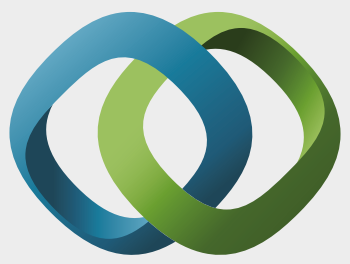

\section{Hindawi}

Submit your manuscripts at

https://www.hindawi.com
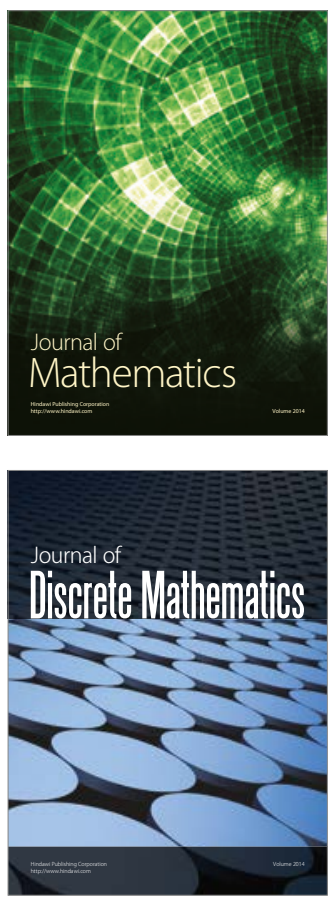

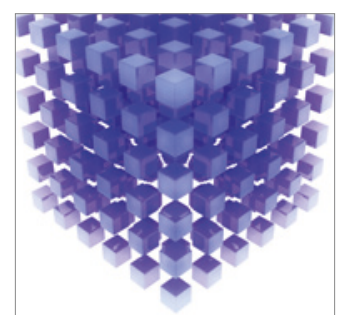

Mathematical Problems in Engineering
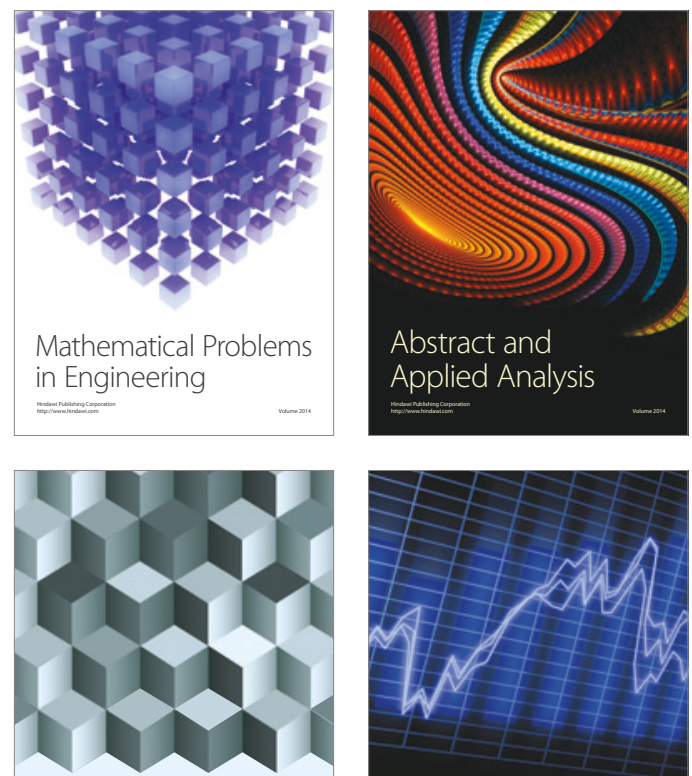

Journal of

Function Spaces

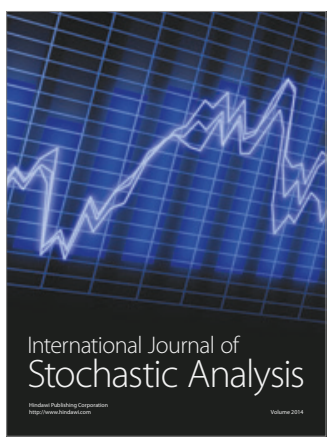

Probability and Statistics
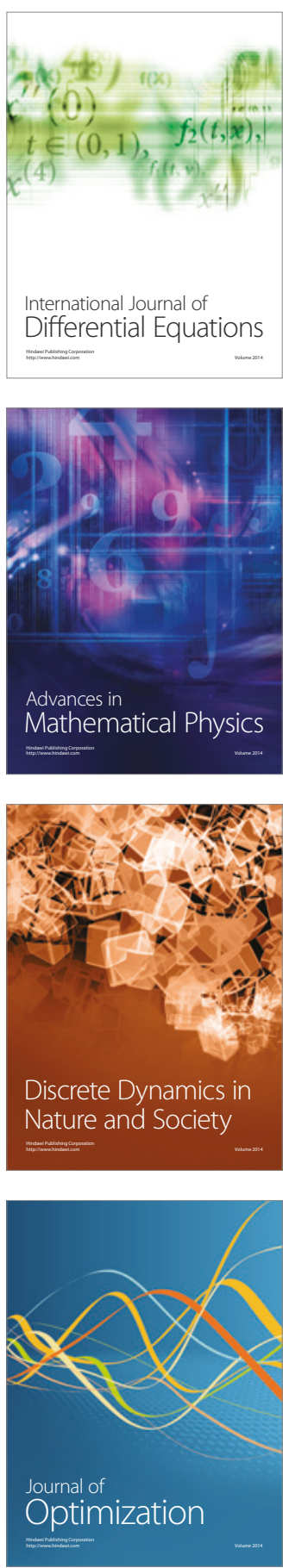